haltigkeitsforschung Dort werden ähnliche Fragestellungen nach den Ursachen oder der Beeinflussbarkeit von Veränderungsprozessen im Umfeld von Energiewende und Klima- wandel diskutiert. Auch hier plant $\mathrm{MATH}^{+}$enge Kooperationen mit exzellenten Partnern aus den entsprechenden Bereichen.

\author{
Dr. Nataša Djurdjevac Conrad, Prof. Dr. Christof Schütte \\ Zuse-Institut Berlin (ZIB), Takustraße 7, 14195 Berlin \\ natasa.conrad@zib.de,schuette@zib.de \\ Prof. Dr. Rupert Klein \\ Freie Universität Berlin, Institut für Mathematik, \\ Arnimallee 6, 14195 Berlin \\ rupert.klein@math.fu-berlin.de
}

\begin{abstract}
Nataša Djurdjevac Conrad leitet die Arbeitsgruppe „Computational Humanities“ am Zuse-Institut Berlin (ZIB) und ist Projektleiterin in MATH ${ }^{+}$. Sie forscht im Rahmen des $M A T H^{+}$Emerging Fields 5 - Concepts of Change in Historical Processes.

Christof Schütte ist Professor für Scientific Computing an der FU Berlin und Präsident des Zuse-Instituts Berlin, wo er den Forschungsbereich "Mathematik für Lebens- und Materialwissenschaften“ leitet. Er ist einer der beiden stellvertretenden Sprecher von MATH ${ }^{+}$.
\end{abstract}

Rupert Klein ist Professor für Scientific Computing an der FU Berlin und Leiter der Arbeitsgruppe „Geophysical Fluid Dynamics“ am Fachbereich Mathematik. Er ist Mitglied des Vorstands und einer der 25 PIs von MATH ${ }^{+}$.

\title{
Tropische Geometrie, lineare Optimierung und Netzwerke
}

\author{
Michael Joswig
}

Das erste Thematic Einstein Semester von $\mathrm{MATH}^{+}$wird im Sommer 2019 stattfinden und den Titel „Network games, tropical geometry and quantum communication" tragen. In diesem Rahmen sind speziell konzipierte Vorlesungen geplant, die kombiniert werden mit Projekten, Vorträgen und Workshops. Höhepunkt des Semesters ist eine internationale Tagung, die vom 3. bis zum 9. Juni 2019 in Berlin stattfinden wird. Das Ziel ist es, drei für sich bereits interessante und umfangreiche Gebiete thematisch zu bündeln und Schnittstellen auszuloten. Die interessanten Anknüpfungspunkte zwischen Quantenkommunikation und Graphenthorie sollen hier nicht vertieft werden. Statt dessen beschränkt sich dieser Text darauf, exemplarisch zu motivieren, wie man von der Geometrie zu Graphen und Netzwerken kommt.

Bereits 2009 schrieben David Speyer und Bernd Sturmfels [10] "... the tropical approach in mathematics ... [is] now an integral part of geometric combinatorics and algebraic geometry. It has also expanded into mathematical physics, number theory, symplectic geometry, computational biology, and beyond." Angefangen hat diese Erfolgsgeschichte mit einem Zählproblem, das in Maxim Kontsevichs Arbeiten zu Gromov-Witten-Invarianten symplektischer Mannigfaltigkeiten auftritt [6]. Grigory Mikhalkin hatte erkannt, dass sich die komplexen algebraischen Kurven im ursprünglichen Problem durch tropische Kurven ersetzen lassen [8].
Letztere haben eine sehr viel einfachere Struktur, enthalten aber immer noch die wesentliche Information. Hannah Markwig hat hierüber schon einmal in den Mitteilungen berichtet [7]. Was ist der Trick?

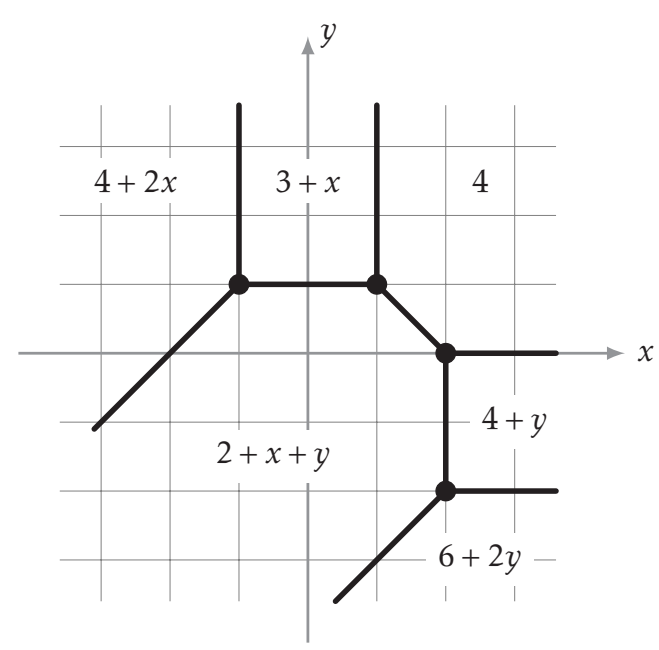

Abbildung 1. Ebene tropische Quadrik zu $F(x, y)$ in (2). Jede Region ist markiert durch denjenigen Term, an dem das Minimum angenommen wird. Die tropische Kurve liegt quasi „dazwischen“, also dort wo dieses Minimum mehrfach angenommen wird. 
Betrachten wir beispielsweise das Polynom

$$
\begin{aligned}
f(x, y)=t^{4}-t^{3} x+\left(t^{4}-2 t^{5}+18 t^{9}\right) y \\
+t^{4} x^{2}+\left(t^{2}+3 t^{3}\right) x y+2 t^{6} y^{2}
\end{aligned}
$$

in den beiden Unbestimmten $x$ und $y$. Die Koeffizienten sind Potenzreihen in einer weiteren Unbestimmten $t$. Durch Tropikalisierung entsteht die stückweise lineare Funktion, die das Paar reeller Zahlen $(x, y)$ auf

$$
F(x, y)=\min (4,3+x, 4+y, 4+2 x, 2+x+y, 6+2 y)
$$

abbildet. Hierbei wird die Multiplikation in (1) durch die Addition ersetzt, sowie die Addition durch das Minimum, die Koeffizienten werden durch die jeweils kleinsten Exponenten von $t$ ausgetauscht. So entsteht etwa aus dem Term $\left(t^{2}+3 t^{3}\right) x y$ die affine Linearform $2+x+y$. Dieselbe Linearform entstünde beipielsweise auch aus $\left(-17 t^{2}+t^{14}-2 t^{15}\right) x y$; insbesondere spielt das Vorzeichen des Koeffizienten der kleinsten $t$-Potenz, 1 bzw. - 17, hier keine Rolle. Das Polynom $f(x, y)$ definiert eine ebene algebraische Kurve über dem Körper $\mathbb{C}\{t\}$ der Puiseuxreihen mit komplexen Koeffizienten, eine Quadrik. Analog liefert das tropische Polynom $F(x, y)$ eine ebene tropische Kurve. Diese Kurve ist die Menge derjenigen Punkte, an denen das Minimum mehr als einmal angenommen wird; siehe Abbildung 1. Durch je fünf Punkte in der Ebene (in allgemeiner Lage) geht genau eine klassische Quadrik. Für tropische Quadriken gilt genau dasselbe. Ebenso geht durch zwei verschiedene Punkte genau eine klassische Gerade und (im generischen Fall) auch genau eine tropische Gerade. Das oben erwähnte Zählproblem von Kontsevich-Mikhalkin betrifft die Verallgemeinerung auf ebene Kurven beliebigen Grades. Der Umstand, dass es keinen Unterschied macht, ob man das Problem klassisch oder tropisch betrachtet ist ein Beispiel dafür, wie Tropikalisierung wesentliche Information erhält. So werden nichtlineare algebraische Daten durch stückweise lineare planaren Graphen codiert. Unter anderem liefert dies unmittelbar Algorithmen [3].

Tropikalisierung ist jedoch nicht beschränkt auf algebraische Kurven oder allgemeinere algebraische Varietäten. Tatsächlich gibt es eine mehrere Jahrzehnte zurückreichende Tradition der „(min,+)-Algebra“ in der Optimierung. Eine elementare aber nützliche Beobachtung ist zum Beispiel, dass die Berechnung der kürzesten Wege zwischen je zwei Knoten in einem endlichen gerichteten Graphen äquivalent ist zu einer Rechnung im Halbring der Matrizen mit (min, +)-Arithmetik. Eher neu ist es dagegen, die dahinterliegenden algebraisch-geometrischen Ideen für Optimierung und Komplexitätstheorie nutzbar zu machen.

Auf Steve Smale's Liste von mathematischen Problemen für das 21. Jahrhundert [9] taucht als Nummer 9 die Frage auf, wie schnell man lineare Programme lösen kann. Zwar hatte Khachiyan bereits 1979 gezeigt, dass es einen polynomiellen Algorithmus gibt, der die Zulässigkeit eines linearen Programms entscheidet [5]. Aber es ist offen, ob das auch dann in Polynomialzeit geht, wenn man die arithmetische Komplexität nach Einheitskosten bemisst, also unabhängig von der binären Codierungslänge der Operanden. Hierzu gibt es nun Fortschritte, die sich der tropischen Geometrie als Methode bedienen.

Statt des algebraisch abgeschlossenen Körpers $\mathbb{C}\{t\}$ betrachtet man den zugehörigen reell abgeschlossenen Teilkörper $\mathbb{R}\{t\}$ der Puiseuxreihen mit reellen Koeffizienten. Der trägt eine natürliche Anordnung, und zwar durch genau das Vorzeichen der $t$-Potenz kleinster Ordnung, das wir bei der Tropikalisierung oben zunächst ignoriert hatten. So erhält man nun Ungleichungen, konvexe Polyeder und lineare Programme über $\mathbb{R}\{t\}$. Damit lassen sich drei verschiedene Sorten von geometrischen Objekten zueinander in Beziehung setzen: Polyeder über $\mathbb{R}\{t\}$, ihre Tropikalisierungen und gewöhnliche Polyeder über $\mathbb{R}$; letztere gewinnt man durch Einsetzen in formale Puiseuxreihen (wobei man sich natürlich Gedanken um die Konvergenz machen muss). In [2] wurde dieser Ansatz genutzt, um erstmals relevante Versionen der Methode der inneren Punkte von einer positiven Lösung des neunten Smale-Problems auszuschließen. Dies ist insofern bemerkenswert als diese Algorithmen durchaus eine polynomielle Laufzeit im Bitkostenmodell haben [4]. Die Idee besteht darin, zunächst eine Familie tropischer Polyeder bzw. tropischer linearer Programme zu konstruieren, diese zu linearen Programmen über $\mathbb{R}\{t\}$ zu liften, um schließlich durch Einsetzen klassische lineare Programme über $\mathbb{R}$ zu bekommen. Die totale Krümmung des zentralen Pfads dieser linearen Programme ist extrem hoch, und dies geht damit einher, dass diese linearen Programme mit der Methode der inneren Punkte schwer zu lösen sind. Wie bei den Kurven sind auch die tropischen linearen Programme einfacher zu analysieren, enthalten aber die entscheidende Information.

Vielleicht kommt aber die Lösung für Smales neuntes Problem aus speziellen Netzwerkspielen. Um dies zu beleuchten sehen wir uns in (3) ein Beispiel für ein System linearer Ungleichungen über $\mathbb{R}\{t\}$ an und seine Tropikalisierung (4):

$$
\left\{\begin{aligned}
t+6 y & \geq 4 x \\
x & \geq 4 t^{4}+5 t^{2} y \\
y & \geq 4 t^{3}+7 t x \\
4 & \geq x \\
3 & \geq y
\end{aligned}\right.
$$

Eigentlich funktioniert die Tropikalisierung termweise wie beim Übergang des Polynoms (1) über $\mathbb{C}\{t\}$ zum tropischen Polynom (2). Jedoch muss man die linearen Ungleichungen zuerst so schreiben, dass auf beiden Seiten der Ungleichung nur positive Terme auftreten; nach der Tropikalisierung dreht sich außerdem die Orientierung der Ungleichung um. Für ein System tropischer linearer Ungleichungen kann man nun wieder die Frage nach der Zulässigkeit stellen, d. h., ob es einen Punkt gibt, der alle Ungleichungen simultan erfüllt. Marianne Akian, Stéphane Gaubert und Alexander Guterman [1] haben gezeigt, dass dieses Entscheidungsproblem polynomial äquivalent ist zu dem bekannten Entscheidungsproblem MEAN-PAYOFF. Hier wird aus den Ungleichungen ein endlicher gerichteter bipartiter Graph konstruiert mit 

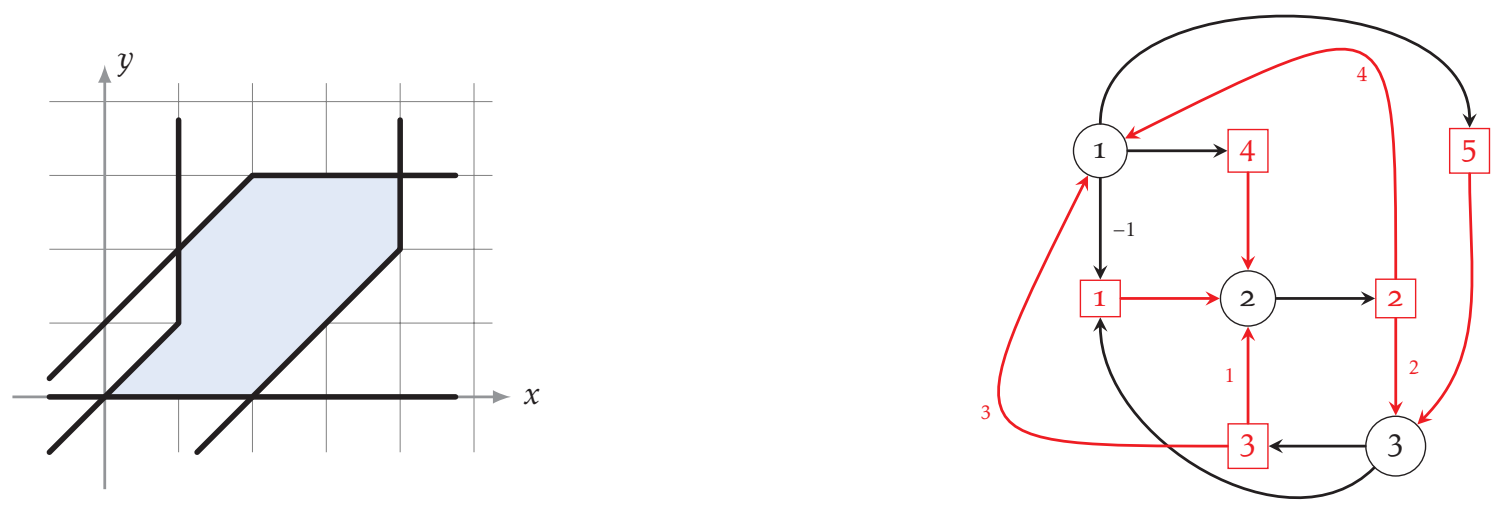

Abbildung 2. Links: ebenes tropisches Polyeder definiert durch (4). Rechts: Graph des zugehörigen Mean-Payoff-Spiels. Die unmarkierten Kanten haben Gewicht 0.

den Koeffizienten der tropischen linearen Ungleichungen als Kantengewichten; siehe Abbildung 2. Auf diesem Graphen ziehen zwei Spieler abwechselnd einen Chip von Knoten zu Knoten entlang gerichteter Kanten. Gespielt wird unendlich lang, und der Ertrag ist das aufsummierte Kantengewicht, gemittelt über die Pfadlänge. Das Vorzeichen des Ertrags signalisiert, wer gewinnt. Das Entscheidungsproblem MEAN-PAYOFF fragt nun danach, wer von den beiden Spielern eine Gewinnstrategie hat. Die Menge der zulässigen Punkte zu (4) und das zugehörige Spiel sind in Abbildung 2 dargestellt. Auf allen drei durch einen Kreis markierten Knoten kann man beginnen, und erhält jeweils einen im Mittel nicht-negativen Ertrag; das heißt der anziehende Spieler gewinnt stets. Dem entspricht, dass (4) (sogar mehr als) eine zulässige Lösung in $\mathbb{R} \cup\{\infty\}^{2}$ besitzt, deren (homogene) Koordinaten alle endlich sind.

Das Entscheidungsproblem MEAN-PAYOFF ist von Bedeutung, weil man weiß, dass es einerseits in den Komplexitätsklassen NP und co-NP liegt, andererseits aber kein Polynomialzeitalgorithmus bekannt ist. So führt die Methode der Tropikalisierung, die zunächst mit algebraischen Kurven begonnen hat, richtig angewendet auf Systeme linearer Ungleichungen zu Netzwerkspielen, die sich im direkten Umfeld der Frage „P $=N P$ ?" befinden. Die Beantwortung dieser Frage ist bekanntlich eine Million Dollar wert und übrigens das dritte Problem auf Smales Liste [9].

Graphen und Netzwerke treten also natürlich auf, wenn man die tropische Methode auf algebraische Kurven oder konvexe Polyeder anwendet. Manch tiefere Frage, wie Smales neuntes Problem, führt dann auf spieltheoretische Aspekte. Auch den Kurven ist das Spielen übrigens nicht fremd: Divisorenklassen auf tropischen Kurven lassen sich durch ein Netzwerkspiel studieren, das chip-firing genannt wird. Im Thematic Einstein Semester „Network games, tropical geometry and quantum communication" soll etwa nach Be- ziehungen geforscht werden zu Netzwerkspielen, mit denen Gleichgewichtssituationen in der Ökonomie untersucht werden. Dies gilt ebenso für interessante Fragen über Graphen und Netzwerke, die sich aus der Quantenkommunikation ergeben. Aber das ist eine andere Geschichte.

\section{Literatur}

[1] Marianne Akian, Stéphane Gaubert, and Alexander Guterman, Tropical polyhedra are equivalent to mean payoff games, Internat. J. Algebra Comput. 22 (2012), no. 1, 1250001, 43, doi:10.1142/So218196711006674. MR 2900854

[2] Xavier Allamigeon, Pascal Benchimol, Stéphane Gaubert, and Michael Joswig, Log-barrier interior point methods are not strongly polynomial, SIAM J. Appl. Algebra Geom. 2 (2018), no. 1, $140-178$.

[3] Simon Hampe and Michael Joswig, Tropical computations in polymake, Algorithmic and experimental methods in algebra, geometry, and number theory (Gebhard Böckle, Wolfram Decker, and Gunter Malle, eds.), Springer, Cham, 2017, pp. 361-385. MR 3792732

[4] Narendra K. Karmarkar, A new polynomial-time algorithm for linear programming, Combinatorica 4 (1984), no. 4, 373-395.

[5] Leonid G. Khachiyan, A polynomial algorithm in linear programming, Dokl. Akad. Nauk SSSR 244 (1979), no. 5, 1093-1096. MR 522052

[6] Maxim Kontsevich, Intersection theory on the moduli space of curves and the matrix Airy function, Comm. Math. Phys. 147 (1992), no. $1,1-23$.

[7] Hannah Markwig, Tropische Geometrie, Mitteilungen der DMV 18 (2010), no. 2, 80-83.

[8] Grigory Mikhalkin, Enumerative tropical algebraic geometry in $\mathbb{R}^{2}$, J. Amer. Math. Soc. 18 (2005), no. 2, 313-377. MR 2137980 (2006b:14097)

[9] Steve Smale, Mathematical problems for the next century, Math. Intelligencer 20 (1998), no. 2, 7-15. MR 1631413

[10] David Speyer and Bernd Sturmfels, Tropical mathematics, Math. Mag. 82 (2009), no. 3, 163-173. MR 2522909

Prof. Dr. Michael Joswig

Technische Universität Berlin, Institut für Mathematik, MA 6-2,

Straße des 17. Juni 136, 10623 Berlin

joswig@math.tu-berlin.de

Michael Joswig ist Einstein-Professor für Diskrete Mathematik/Geometrie an der TU Berlin. Gemeinsam mit Jens Eisert und Max Klimm organisiert er das Thematic Einstein Semester „Network games, tropical geometry and quantum communication“. 